Article

\title{
The Indirect Perception of Distance: Interpretive Complexities in Berkeley's Theory of Vision
}

Michael James Braund

In truth and strictness of speech I neither see distanœe itself, nor anything that I take to be at a distanoe. I say, neither distance nor things plaoed at a distanoe are themselves, or their ideas, truly perceived by sight. $\mathrm{G}$ eorge B erk eley ${ }^{1}$

$\mathrm{T}$

he problem of whether perception is direct or if it depends on additional, cognitive contributions made by the perceiving subject, is posed with particular force in an E ssay towards a $\mathrm{N}$ ew Theory of $\mathrm{V}$ ision (N TV ). It is evident from the recurrent treatment it receives therein that Berkeley considers it to be one of the central issues concerning perception. Fittingly, the NTV devotes the most attention to it. In this essay, I deal exclusively with Berkeley's treatment of the problem of indirect distance perception, as it is presented in the context of that work. This task will consist of three parts.

In Section One, I provide an outline of Berkeley's answer to the question, "What are we immediately aware of in perceptual experience?" Here, I will pay particular attention to Berkeley's use of the term "association" in describing the indirect or mediate procedure whereby vision acquires information about the spatial layout of the external world. Accordingly, it will be shown that while the visual perception of space is a derivative process, "seeing" distance is by no means a matter of performing geometrical inferences (as D escartes contends). Rather, through an ongoing encounter with stimuli in the environment, perceivers develop the habit of associating one order of sensory information with another. Since Berkeley thought that visual experience lacked inherent three-dimensionality, and that "distance" was registered only by the sense of touch, he hypothesized that retinal information acted as a cue for tangible consequences in the environment. The result is what Robert Schwartz calls "the pragmatic significance of vision," which is "essentially a guide to movement and touch." ${ }^{2}$ For Berkeley, distance

1 G. Berkeley, A n E ssay Towards a N ew Theory of V ision (New York: Bobbs-Merrill Company Inc., 1963), 39.

${ }^{2}$ R. Schwartz,V ision: V ariations on some Berkelean Themes (Oxford and Cambridge U.S.A.: Blackwell Publishers, 1994), 9.

(c) 2007 Michael James Braund http:// www.kritike.org/journal/issue_2/braund_december2007.pdf ISSN 1908-7330 


\section{THE INDIRECT PERCEPTION OF DISTANCE}

perception is intimately related to movement and to the guidance and adjustment of behavior. Moreover, I will show that this capacity for associating two-dimensional visual information with three-dimensional tactile data is precisely what enables Berkeley to maintain an indirect theory of distance perception, while rejecting Descartes's geometrical solution. In Section Two, I will examine Berkeley's explicit rebuttal of inferential accounts and outline his critique of geometrical optics (sections 3-15 in the NTV). Thereafter, I will consider his conclusions concerning the derivative nature of distance perception and the heterogeneity of the ideas of sight and touch regarding individual objects. In Section Three, I will draw on the information provided by the previous sections to arrive at a more robust conception of Berkelean "associations." I will characterize the logic underlying "associations" as indudivein nature, and then formally contrast them with D escartes's deductive, geometrical "inferences."

Since Berkeley's terms are challenging, and in some cases forbiddingly technical, I will begin by clarifying his key concepts. Unlike most of Berkeley's commentators, however, I will perform this task in the order of their original composition. "Like all of Berkeley's writings, the New Thery of V ision has a clearly defined organization." 3 Its expression is deliberate and methodical, and demands to be read in a particular order. Therefore, I have chosen to begin with Berkeley's own, carefully selected starting place. I will follow along with Berkeley and bring to light those premises from which his seemingly fantastic conclusions are drawn.

\section{Section 1: Mediate and Immediate Perception in the NTV}

For such a shrewd theoretician, it is strange that Berkeley begins as uncritically as he does. In section 2 of the NTV, he states, "It is, I think, agreed by all that distance, of itself and immediately, cannot be seen." 4 The fact that Berkeley did not see the need to explain this phrase, or to provide arguments in support of it, might in all propriety be used as a criticism against him. ${ }^{5}$ In any case, this initial statement lays down the terms of a problem

\footnotetext{
${ }^{3}$ D.M. Armstrong, D.M., Bekkdeys Theary of Vision (Australia: Melbourne University Press, 1960), 1

4 G. Berkeley, An Essay Tonards a New Theary of Vision (New York: Bobbs-Merrill Company Inc., 1963), 19.

5 However, it is my suggestion that this statement can be read to reflect the epistemological climate of Berkeley's day. As a young man of twenty-four, Berkeley composed the NTV with the looming presence of his fellowship examination in mind. Accordingly, he wrote his work on vision as a formal study of contemporary issues in the philosophy of perception; and by its first publication in 1709, nothing was more contemporary in European intellectual life than the work of D escartes. D escartes, a great champion of indirect perception, was the most influential student of vision since Kepler; his works were published in three languages and accepted as the "received" view on all fronts. Since Berkeley was addressing a committee of Cartesians, he chose to work within a Cartesian context, stressing the derivative nature of distance perception. The view that perception is indirect was so often conceded in the $18^{\text {th }}$ century, that Berkeley willingly admitted it as the first principle of his NTV.
} 
about distance perception that he regards as widely recognized. Since the whole argument of the NTV depends upon this contention, it deserves careful scrutiny.

According to Armstrong, the word "distance" presents no problems. It merely refers to the line directed endwise to the eye, at right angles to the retina (sometimes called "outness" by Berkeley). Rather, it is the phrase "immediately seen" that gives us trouble, and so we shall dismiss three false interpretations from the start.

(1) When Berkeley says that the perception of distance by sight is a "mediate" procedure, his claim is not the obvious one that naked vision makes less-than-exact estimates of distance. To cite G.J. Warnock's example, someone might say, "There is a gap between object A and object B." To this I might correctly agree. However, if they had said, "The distance between object A and object B is exactly $\mathrm{x}$ meters and y centimeters," then I would be incorrect in saying that this is what I had perceived. While I might claim to have guessed that the distance between the two points was something in that range, I could not claim to seethat it was exactly that. After all, nobody sees distance with a surveyor's accuracy. ${ }^{6}$

In any event, this is not Berkeley's point. His point, rather, is the more extreme one that (unmediated) vision is altogether incapable of making estimates about distance. This contention is meant to cover even the most rough and ready approximations. For Berkeley, we cannot properly be said to "see" the distance of objects. This is because "distance, being a line directed endwise to the eye ... projects only one point in the fund of the eye, which point remains invariably the same, whether the distance be longer or shorter." 7 This is called the "one-point argument" and is important, because it forms the basis of his theory indirect perception. Essentially, the argument states that a point at any distance along a line of sight projects only a single point on the retina. The light striking this point could be from an object at any distance at all (i.e., one meter, one hundred meters, two hundred meters, and so on). In each case, the spatial extent between the object and our eye is not represented anywhere on the retina. Retinal information, ${ }^{8}$ which is to say, the global pattern of light stimuli incident on the retinal plane, does not specify the tridimensionality of the perceptible world. "In a strict sense," Berkeley writes, "I see nothing but light and colors with their several shades and variations." 9 So even though distance appears to be seen immediately along the line of vision, vision as surhis without a sense of depth.

(2) This latter claim faces a possible, albeit wrongheaded objection. That things look to be sitting out there, independent of the perceiver, is a characteristic of the visual field. O ne might, therefore, be inclined to reject Berkeley's contention on the ground that it seems to deny the obvious fact that

${ }^{6}$ G J. Warnock, G J., Bekkey (Middlesex England: Penguin Books, 1969), 26.

7 Berkeley, An Essay Tovardsa NewTheary of Vision 19.

${ }^{8}$ Which Berkeley regarded as the only information exclusive to vision.

9 Berkeley, AnEssay Tonardsa NewTheryof Vision 82. 


\section{THE INDIRECT PERCEPTION OF DISTANCE}

things really do look distant. We often say "It looks a very long way off" or "I saw him in the distance." In these cases, we claim to see things that are at distances from us, and more importantly, that look as if they are. Therefore, if Berkeley's theory denies this common sensible claim, then he really is trying to paddle up-stream. And while "common sense" is by no means the measure of a philosophical theory, ${ }^{10}$ it certainly holds weight when that theory purports to talk about everyday experiences, like seeing things "in the distance."

This is the most typical misunderstanding of Berkeley's theory (i.e., that he wants us to accept premises about experience that conflict with it). In response, I need only point to a passage from The Principles of $\mathrm{H}$ uman Knowledge: Berkeley writes, "That we should in truth see external space, and bodies actually existing in it, some nearer, others farther off ... gave birth to my E ssay towards a $\mathrm{N}$ ew Theory of $\mathrm{V}$ ision, which was published not long since." 11 The central concern of the NTV is to explain how objects appear in visible space. That they appear in visible space is a fact so obvious, that Berkeley fails to even mention it. Similarly, the "flat image" problem arises, not because the visual field looks to be two-dimensional, but because it does not look twodimensional. If it did, if the visual field had the same non-spatial organization as the retinal image, then there wouldn't be a problem in the first place. Accordingly, the main thrust of the N TV is to explain how objects in the visual field acquire distance qualities. ${ }^{12}$

(3) Another objection to Berkeley's thesis may arise if, by the term "immediacy," one imagines a "temporal immediacy." For instance, no sooner have I opened my eyes, than I am immersed in a world of light, colors and textures. D istances trace themselves out around me and things autonomously jut out in three-dimensions. It would seem, on this objection, that distance must be immediately perceptible by sight. That is, distance is perceived at the same time that light, color, and form are perceived.

However, this reply misses the mark. Berkeley is not employing "immediacy" in any temporal sense of the word. Besides, there is nothing in the NTV to suggest that we cannot make visual estimates of distance at a glance. The process whereby distance is suggested to the eye is as temporally immediate as the perception of light and color is. "I can see that the tree is more distant than the man, just as immediately as I can see that it is to the left of the man, or that its leaves are green." 13 Thus, to get at Berkeley's claims about distance perception, the term "immediate" will have to be clarified.

What is an immediate perception? According to Berkeley, immediate perceptions are purely sensorial, "non-mental goings-on."14 A perception can

10 Berkeley does, however, make "common sense" a necessary condition of philosophical theories in the later writings, such as the Principles (1710) and the Three Dialogues (1713). Inc., 1963), 3-4.

${ }^{11}$ G. Berkeley, The Principles of $\mathrm{H}$ uman Knowledge (New York: Bobbs-Merrill Company

12 I will return to this in Section Two.

${ }^{13}$ Armstrong, op cit., 3.

${ }^{14}$ Schwartz, op cit., 10. 
be called "immediate" if it is not the result of a process that has mental or psychological components. In this sense, "The processes that underlie immediate perceptions are comparable to those that underlie the output of our kidneys or ... are responsible for our blinking when air is puffed in our eyes." 15 For D escartes and Berkeley, they are entirely physiological in nature, involving nothing that could be called a mental operation. "Mediate perceptions," on the other hand, are of mental origin; and not only that, "but one or more of the intermediate stages leading to our having the idea ... has ideational or mental content." 16 In the case of distance perception, then, ideas of distance are suggested to vision by means of some other intermediate ideas. For Berkeley, these intermediate ideas are "immediately perceived in the act of vision." 17 For example, light rays, along with certain movements and adjustments of our eyes, cause us to have an array of sensations, which in turn bring it about that we experience an idea of distance. The initial sensory array is immediate. It is the result of purely physiological operations, whereas our idea of distance is nonimmediate, depending as it does on the immediate sensory array to bring it about. Therefore, the claim that "we do not see distance immediately" amounts to the claim that the ideas of distance, derived from sight, depend on mental operations. For Berkeley, this means that ideas of distance are brought to mind via intermediate ideas. To clarify this, I will consider three of Berkeley's more instructive examples from the N TV .

(1) In the first case, Berkeley compares his model of distance perception to his theory of language comprehension. Berkeley's philosophy of language is remarkably similar to Locke's. In order to understand someone's speech, two things must occur. First, we must hear what the person says. It is the experience of hearing the word spoken that triggers the appearance of the idea that the word represents. For example, the idea, "coach," that comes to mind is not immediate; rather, it is the result of our having an intermediate idea. In this case, the intermediate idea is the auditory (or visual) experience of hearing (or reading) the word "coach." Berkeley theorizes that we do not focus attention on these intermediate ideas and may not be readily aware that we are even having them. It is as if we "read through the experience or sensation of the sign when our attention is directed to its significance."18 What concerns us is the ideational content, the "coach" idea, not its sign. Moreover, in most cases of developed space perception, the process of an intermediate idea triggering a mediate idea is automatic; there is an atemporal leap from the one order of perception to the other. Berkeley writes, "No sooner do we hear the words of a familiar language pronounced in our ears but the ideas corresponding thereto present themselves to our minds; in the very same instant the sound and the meaning enter the understanding" 19 (my italics). Thus, it may

\footnotetext{
15 Ibid.

16 Ibid.

17 Berkeley, A n E ssay T owards a N ew Theory of V ision, 22.

18 Schwartz, op cit., 10.

19 Berkeley, A n E ssay T owards a N ew Theory of V ision, 42.
} 


\section{THE INDIRECT PERCEPTION OF DISTANCE}

be impossible to introspectively isolate the qualities of the intermediate sensation for singular investigation.

(2) To better illustrate Berkeley's meaning, consider a second analogy from the NTV. Berkeley writes, "Sitting in my study I hear a coach drive along in the street; I look through my casement and see it; I walk out and enter into it." 20 In ordinary discourse, he says, we would be inclined to think that we hear, see and touch the same thing, namely, the coach. However, there is also some important sense in which we cannot hear the coach, but only the sound that it makes.

Berkeley is pointing to a real distinction here. He explains that hearing the misea coach makes, requires only that our auditory sense faculty be in good working order. Conversely, hearing the coachitself requires that a "background of experience" 21 be presupposed. In this case, the background is made up of past associations between auditory stimuli in the environment and other types of stimuli, the combination of which specifies the object "coach" with certain stimuli and does so with regularity. Consequently, the auditory stimulus, which contains all of the information proper to hearing, is insufficient to account for a perception of the coach. The coach can only be heard on the condition that the auditory data is supplemented by additional kinds of sensory information (i.e. visual, tactile, etc.). This process of supplementation is really at the heart of what Berkeley calls "mediate," as opposed to "immediate" perception.

Thus, an immediate perception is a perception that depends upon no other. It requires only the vigor of our sense faculties and the presence of stimuli in the environment. Mediate perception, by contrast, draws on a much broader sense of the verb "to hear". It involves, not so much a deductive inference, as an experiential association of the mind. In Section Three, I will return to this distinction and highlight the differences between them.

(3) Another way of making clear this distinction draws on the "argument from illusion." Sensory illusion occurs when the immediate object of perception is either (a) not actually as it appears to us, or (b) is simply unreal. For instance, if a man suffers from jaundice, whatever he perceives will seem to have a yellow tinge about it. Since what he perceives is not actually yellow, but only appears yellow, we say that this man has been subject to a sensory illusion. Similarly, if I believe I have heard a sound, when in fact there is no such sound to be heard, then I have fallen victim to sensory illusion. On the other hand, if I believe that the sound I have heard is a coach, when in fact it was a hay wagon or a dog sled, I need not have been subject to illusion. This is because it might be that other objects besides coaches make that noise, and, really hearing the noise, I misjudge that there was a coach in the street. Therefore, whereas sensory illusion applies in the case of immediate perception, it does not hold at the mediate level.

This example should clarify the distinction Berkeley has in mind. More to the point, this distinction applies equally in the case of vision. When

\footnotetext{
${ }^{20}$ Ibid, sec. 40.

${ }^{21}$ Armstrong, quit, 3.
} 
Berkeley claims that "distance, of itself and immediately, cannot be perceived by sight," what he means to say is that distance is itself not a proper object of sight. Rather, three-dimensional vision is the outcome of mediate procedures. It depends, for its existence, upon a series of habitual associations that link sensory-retinal information (i.e., light, color and form) together with tactile information about distance. On this account seeing distance is always like hearing coaches, and never like hearing sounds.

Berkeley's solution to the problem of distance depends, in part, upon his refutation of the received view. If his theory succeeds, it does so on the condition that he can give a better account than his predecessors have. Therefore, I will turn toward Berkeley's proposed target: the geometrical theory of space perception.

\section{Section 2: Berkeley's Refutation of the Geometric Theory of Perception}

In section two of the NTV, Berkeley is doing nothing more than pointing to a problem he takes to be well understood. He did not think that this point was original or controversial. In fact, there are passages in Molyneux's Nova Diqptica, which suggest that section two of the NTV is simply paraphrased from this earlier work. D escartes himself entertained this notion in the Diqptrics, as the mechanical processing of light stimuli encoded as motion. In this section, I will address the main claims in Descartes's geometrical theory of space perception, and will discuss Berkeley's critique thereof.

As Warnock points out, "Berkeley saw clearly, as too many writes on the 'theory of vision' did not, that questions of several different kinds can be asked about seeing, and that serious mistakes will be made unless each kind is properly distinguished from the others." 22 For instance, some questions about vision pertain exclusively to the geometry of optics. These include questions about refraction, reflection, magnification, and the focal length of lenses. To this end, the eye does behave like an adjustable lens. However, these questions that can be posed and answered in purely geometrical terms, concern "the mechanism of the eye," and therefore, "appertains to anatomy and experiments." 23 While the doctrines of this study are no doubt valid and theoretically useful, they are of a completely different kind than Berkeley's field of study. Berkeley is interested in the manner by which we perceive, via sight, the distance of objects in space. This latter investigation is, by contrast, of purely psychological value. It studies the experience of space perception and the equally experienceable conditions that make it possible (I will explain this momentarily). Answering questions about psychology by appeal to geometry is a typical example of a category mistake; that is, of ascribing the features of

22 Warnock, qpit, 26.

${ }^{23}$ G . Berkeley, The Theory of V ision Vindicated (N ew York: Bobbs-Merrill Company Inc., 1963), 139 


\section{THE INDIRECT PERCEPTION OF DISTANCE}

something to one category, which are only attributable to another. While this is not the only dimension of Berkeley's critique, it is an important aspect of it. This partition, separating geometrical and psychological optics is among Berkeley's greatest advancements in the science of vision. Berkeley criticizes D escartes for failing to make the same distinction. However, there is still much to be said about Berkeley's critique of Cartesian optics.

A point of commonality between Descartes and Berkeley is their use of the one-point argument. In view of this argument, many writers on optics found it increasingly difficult to explain how perceivers estimate the distance of objects in space. The orthodox view, of which Descartes was a leading proponent, attempted to solve the difficulty by appeal to the fact that most people have two eyes. If both eyes are directed at an object, the two straight lines from the eyes to the object (the 'optic axes') will converge; and if the object is close enough to the eyes to "bear any sensible proportion" 24 to them, the angle of intersection of the optic axes will be fairly large. In these cases, it was supposed that perceivers were able to calculate the distance of an object in space from the angle at which the optic axes converge upon it; the larger the angle the closer the object.

This theory faces an immediate criticism. Even if we suppose that its claims hold true in cases of binocular disparity, what about those who have to or for any reason only wish to use one eye? After all, if I shut my left eye the world doesn't appear flat - it retains its three-dimensional composure. Moreover, horses and chickens have a fine sense of depth, but their eyes are on opposite sides of their heads. Their visual fields do not overlap. Thus, they are unable to triangulate objects in the Cartesian manner.

The geometrical opticians do make provisions for these cases in which "we see with only one eye at once being exploded." 25 Their argument here was that the rays of light emitted or reflected from an object very close to us must converge quite sharply upon the eye, and that this angle of convergence decreases as the object is moved farther away. In this way, the distance of the object could be worked out from the angle at which the rays converge on the eye. Of course, this model will only work in cases where the object is close enough to the eye; for as it recedes, the angles of convergence soon become so small that we could not reasonably be supposed to detect any further variations within them. With this one restriction, the problem was widely held to have been solved.

With his usual candor, Berkeley rejects the whole of this account; his reason being that "when the mind perceives any idea not immediately and of itself, it must be by means of some other idea," 26 of which the mind is immediately aware. In other words, whatever it is that leads me to form a particular judgment on any occasion must be something of which, on that occasion, I am aware; and I am certainly not aware of lines and angles.

24 Berkeley, A n E ssay T owards a N ew Theory of V ision, 20.

25 Ibid., 20.

${ }^{26}$ Ibid., 21. 
Consider Berkeley's example. He writes that while "the passions which are in the mind of another are of themselves to me invisible. I may nevertheless perceive them by sight; though not immediately, yet by means of the colors they produce in the countenance." 27 So supposing, on meeting with my friend, I got the impression that he was angry. If I were to inventory the observations that led me to my belief, "Paolo is angry," I might say that his cheeks appeared flushed, or that he frowned and furrowed his brow as he spoke. That is why I thought he was angry. Now these qualities of being flushed or frowning, Berkeley says, could never have produced in me the idea, "Paolo is angry," had I not been aware of them in the first place. Moreover, the possible fact that his adrenal medulla was secreting catecholamine (a stress response in the autonomic division of the sympathetic nervous system) could not have contributed to my belief, if either (1) I did not know that this was the case, or (2) I did not know that hypothalamic reactivity had anything to do with being angry. Therefore, Berkeley concludes, circumstances of which I am unaware have no influence over my ideas. ${ }^{28}$

In Berkeley's mind, the geometrical opticians had committed exactly this kind of mistake. Their statements about lines and angles might be correct, and of great theoretical value, but it is obvious that perceivers do not in fact make use of geometry in estimating by sight the distance of objects in space. Berkeley explains, "Those lines and angles, by means whereof some men pretend to explain the perception of distance, are themselves not at all perceived; nor are they in truth ever thought of by those unskillful in optics." 29 The fact of the matter is that we do not see lines and angles running out from our eyes and converging upon an object in space (or vice versa). These lines and angles are to be found in the theorists' diagrams, which are useful in (1) measuring distances, but never in (2) judging them by naked vision alone. Warnock points out that, while the contour lines on a map are useful devices for cartographers, they are useless in helping us to judge the height of hills by vision. ${ }^{30}$ Confusing (1) with (2) is an instance of a category mistake; that is, of confusing theory with reality, or the menu with the meal. While the claims of the geometrical opticians may be true, they cannot be the correct answers to the psychological (qua philosophical) problem proposed.

At this juncture, Berkeley offers his account of distance perception. In sections 1-40 of the NTV, Berkeley argues that the solution to the problem of distance perception will be in virtue of certain "cues," which are effectively available to the visual system. Berkeley admits three cues by which we perceive distance by sight. (1) The first concerns the disposition of theeges wherein, as the object recedes or approaches us, we alter the disposition of the eyes by increasing or decreasing the interval between the pupils. Each disposition is then attended by a sensation, which, by means of an associative connection

27 Ibid

${ }^{28}$ I have adapted this point from G.J. Warnock's book on Bekdey.

29 Berkeley, An EssayTowardsa NewThearyof Vision 22.

30 Warnock, qpait, 28. 


\section{THE INDIRECT PERCEPTION OF DISTANCE}

performed by the mind, produces ideas of distance in us. (2) The second cue involves the notion of oallar darity. The nearer an object approaches to us, the more confusedly it is seen. Using this cue, Berkeley attempts to argue in the "Barrovian Case" that confusedness in vision is "a more important cue for distance than is the established view based on the angles the eyes make at the object." 31 (3) Thirdly, Berkeley considers the oular strain perceivers experience in visual perception. By straining the muscles in the eye, the approaching object can be kept less confused when in the line of sight. This kinesthetic sensation "supplies the place of confused vision in aiding the mind to judge of the distance of the object; it being esteemed so much the nearer by how much the effort or straining of the eye in order to distinct vision is greater." 32

What is perhaps most significant is that, for Berkeley, the passage from a given sign or "cue" to its corresponding distance perception involves a background of experience, the conjunction of visual cues with the object appearing at a distance. Unlike the geometrical cues of D escartes, Berkeley's are non-deductive. There is no neessary connetion between the sensation in the muscles of the eye and the distance at which the object purportedly stands from us. "There is only a contingent connection between these phenomena, and the distance of the object seen." 33 Moreover, the connection between visual cues and the distance of objects is discoverable a posterioi. He is insistent on this point, and rightfully so. Berkeley's solution stands, if the geometrical theory falls. Atherton points out that what Berkeley has done, in effect, is to "take cues mentioned by Malebranche, and then point out that they can serve as distance cues without a mathematical or geometrical interpretation." 34 Therefore, Berkeley concludes his critique of geometrical optics (sections 3-15 in the NTV) by undermining the basic premises of Cartesian spatial perception. Berkeley thinks that D escartes and Malebranche wrongly integrate mathematical processes into their account of the visual estimation of distance.

In the final portion of the NTV (section 121-159), Berkeley says he is going to look into the difference between the objects of sight and the objects of touch, and see whether there is any idea common to both senses. From the premises put forward, he writes "it is a manifest conclusion" that "a man no more sees and feels the same thing than he hears and feels the same thing." This is because "we never see and feel one and the same object. That which is seen is one thing and that which is felt is another." 35 Although shocking, this conclusion is a cogent piece of reasoning and follows quite conclusively from what he has premised.

It is quite common that scholars take Berkeley too literally on this point. Despite Berkeley's somewhat awkward articulation of his conclusion, there is nothing in the NTV to suggest that we cannot see and feel the same

\footnotetext{
${ }^{31}$ Armstrong, qpait, 19.

32 Berkeley, AnEssayTonardsa NewTheory of Vision, 26.

${ }^{33}$ Armstrong, quat, 18.

34 M. Atherton, Bekkdeyss Redution in Vision(New York: Cornell University Press, 1990),

35 Berkeley, AnEssay Towardsa NewThearyof Vision, 40-41.
}

84. 
physical object. The coach I see is the coach I touch. Rather, the object that we never see and feel is, to use Berkeley's expression from the Priniples an "object of sense." In other words, Berkeley is simply pointing to the fact that the data provided by one sense modality (i.e., sight) is not equivalent to that provided by any other. This was made clear by the distinction between the mediate and immediate modes of perception, outlined in Section One. It is precisely because distance (a mediate idea) cannot be attributed to immediate seeing or hearing, that we are forced to make the distinction in the first place. If we follow Berkeley on this claim, if all our visual sensations specify twodimensional data, the question becomes "from where do we get our idea of three-dimensions?" As Berkeley states, distance is not immediately apprehended by sight. It must, therefore, be "brought into view by means of some other idea that is itself immediately perceived." 36 Berkeley concludes that the immediate perception of distance is to be found in tactile experience (using "touch" in a broad enough sense to include kinesthetic experiences). For Berkeley, we fed distance, but never seit.

This conclusion has given rise to a multitude of false interpretations and hasty rebuttals. That being the case, the following point is worth repeating. In stressing the derivative nature of distance perception by sight, Berkeley does not claim that we do not see things at a distance from us. As I have argued, Berkeley is not working against common sense per se $\mathrm{He}$ is only making a revision to common speech. That his view should appear to conflict with former is an outcome of the latter. The elasticity of ordinary language needs to be stretched to cover Berkeley's meaning; and so his view often been made the target of a colossal misinterpretation. Berkeley was acutely aware of this risk. He writes, "The difficulty seems not a little increased because the combination of visible ideas has constantly the same name as the combination of tangible ideas wherewith it is connected - which does of necessity arise from the use and end of language." 37 As A therton points out, what Berkeley intends to say is that "distance information is tangible information suggested by what we see." 38 Given the appropriate cues (a species of vision), ideas of touch, together with the information about distance they embody, are suggested to the mind through habitual associations. What is immediately perceived by sight is simply a reliable sign of what, in all probability, we might experience by touch. Distance is traced out by the various motor functions of my body. To say that what we see is at a distance from us means that what we see suggests to our understanding "That after having passed a certain distance, to be measured by the motion of [the] body, which is perceptible by touch, I shall come to perceive such and such tangible ideas which have been usually connected with such and such visual ideas." 39

36 Ibid, 22.

${ }^{37}$ Ibid, 41.

38 Atherton, qpat, 103.

39 Berkeley, AnEssay Towardsa NewThery of Vision 39. 


\section{0}

\section{THE INDIRECT PERCEPTION OF DISTANCE}

Therefore, spatial ideas are concepts of a specific sort, the meanings of which lie in tangible consequences. To know the distance spanning from myself out to the tree, across the yard, is to have ideas about my moving body, and about how many paces it will take to get to the tree. Seeing distance requires only that "environmentally appropriate motor ideas are derived from the flux of visual sensations, whatever these sensations are like qualitatively." 40 Berkeley's approach to vision is, therefore, a pragmatic one. Vision is a guide to movement and touch. The presence of visual sensations allows perceivers to anticipate a second ordering of ideas which are, although experientially univocal, conceptually unrelated. This view is typically expressed as the heterogeneity of the ideas of sight and touch.

In expressing himself this way, Berkeley's point is the very simple one that two-dimensional objects of sight and three-dimensional objects of touch are conceptually (although not experientially) distinct. That is, they refer to non-identical spaces. The fact that these fields seem to overlap is insufficient to establish their identity. It is true that they are almost invariably associated with one another, however, their associations remain merely contingent, nondeductive facts. Rather, it is the closeness of this association, Berkeley thinks, that leads us into thinking that distance is immediately perceived by sight. 41 Just as, upon hearing the words of a familiar language spoken, we cannot help but associate the noises heard with the meanings that habitually accompany them, so the close connection between the objects of sight and touch implies that "distance" will be irrevocably suggested to vision, whether we like it or not.

\section{Section 3: Inferences and Associations as Explanatory Concepts}

While the introduction of the notion of inference as an "explanatory concept" 42 in the theory of vision is attributable to Descartes, it owes its modern articulation to the work of Herman Von Helmholtz. Following Schwartz, I will briefly consider Helmholtz's theory of perceptual inferences in order facilitate an explanation of the differences between Cartesian inferences and Berkelean associations. In particular, I will discuss the inductive reasoning that underlies both Helmholtz's and Berkeley's approaches, and contrast them with D escartes's deductive model of perceptual inferences.

In his Treatise on Physiological Optics, Helmholtz offers an indirect theory of distance perception that is similar to Berkeley's theory. Like Berkeley, Helmholtz argues that three-dimensionality is not an immediate quality of visual experience (owing to the two-dimensional character of the retinal image). $\mathrm{G}$ iven this limitation, they argue, three-dimensionality must be added to visual

${ }^{40}$ Schwartz, op cit., 9.

${ }^{41}$ D rawing, of course, on the notion of temporal immediacy as discussed in Section One.

${ }^{42}$ Schwartz, op cit., 84. 
perception beyond what is conveyed by processes involved in detecting sensory stimulation. In this respect, Helmholtz argued that distance perception is a "multistage process" in which the "sensory mechanisms respond to light stimuli by producing ... visual sensations [on the retina] which have no inherent spatiality." 43 Therefore, an individual must learn to detect distance through the intervention of mediating perceptual processes, because distance is not an immediate quality of retinal sensations.

Helmholtz's theory, with its roots in the Berkelean paradigm, is fundamentally an associational theory. In other words, Berkeley and Helmholtz stress the importance of "learning," or more specifically, "habit formation" in the development of an individual's spatial perception. On their account, we perceive space derivatively. O ur immediate visual experiences serve as sensory "cues" for the mediate perception of space. Habit, formed by past associations, serves to connect our retinal manifold and kinesthetic eye cues (i.e., convergence, accommodation, etc.) with our tangible sensations of space gained from movement and touch. Berkeley and Helmholtz contend that, because the retinal manifold contains no information about tri-dimensionality, the tangible sensations (with which they are associated) give spatial meaning to our immediate visual experiences. Thus, both thinkers provide an explanation of our ability to perceive three-dimensional space by using an associational model.

Like Berkeley, Helmholtz characterizes his associational model as one involving processes of sign interpretation (the "interpretation" being guided by past associations). As outlined in Section O ne, Berkeley argues that we ought to think of these perceptual processes as similar to the processes involved in understanding language. Immediate visual experiences (i.e., retinal "cues"), like words, are mere signs of their meanings. In order to understand their significance, we must learn what they mean through experience. Just as we are not immediately aware of the spatial properties of objects, but only of a retinal manifold that "serves as a sign to trigger the assignment of [these] properties," 44 so we are not immediately aware of the things that words signify. Helmholtz writes, "O ur ideas of things cannot be anything but symbols, natural signs for things which we learn how to use" 45 (my italics). Spatial perception, like the acquisition of a language, must be learned over time. However, once this connection becomes habitual, and the associations are established, we pay little attention to the actual sign when it is encountered. Instead, our mind leaps to the interpretation that the sign initiates. Thus, spatial perceptions are the "experiential meanings" 46 that result from the interpretations we have learned to associate with our immediately experienced sensations.

The critical point of this analogy, for Berkeley and Helmholtz, concerns the logical connection between the sensory or linguistic sign and that

43 Ibid., 87.

44 Ibid., 84.

${ }^{45}$ H. Helmholtz, T reaties on Physiological Optics (New York: D over, 1950), 19.

${ }^{46}$ Schwartz, op cit., 84. 


\section{THE INDIRECT PERCEPTION OF DISTANCE}

which is signified by it. Both contend that the link between the signs and what they stand for is not a neessary connection. Hence, there is no a prioi way of knowing the spatial significance of our immediate visual experiences, prior to the associations we establish between the tangible ideas of distance and the visual distance cues. Crucially, Berkeley's and Helmholtz's distance cues possess a merely contingent connection with the distance of objects in space; a connection that is learned a posterioi "in the same way we discover that smoke is a sign of fire," 47 or that a word is a sign of an object. This is why Berkeley is hesitant to describe the procedure in which the mind derives distance perceptions from sensations as inferential. Cartesian "inferences" are based on deductive principles, which enable the visual system to calculate a percept from the immediate sensory data, "it being a ... neessary truth that the nearer the direct rays falling on the eye approach a parallelism, the further off is the point of their intersection, or the visual point from whence they flow." 48 In contrast, Berkelean "associations" involve a mental synthesis of past and present experiences by which perceivers lem to associate tactile and visual orders of sensory information through experience. For Berkeley, there is no innate or "necessary connection between the sensation we perceive ... and greater or lesser distance." 49 Rather, "because the mind has, by constant experience, found the different sensations corresponding to ... a different degree of distance in the object - there has grown a habitual or customary connection" 50 between immediately perceived retinal sensations and mediately perceived ideas of distance.

Helmholtz expresses the same point in different terms. In the chapter "Concerning the Perceptions in General" Helmholtz stresses the analogy between the associative processes underlying vision and those used in ordinary indudive reasoning. He claims that associative processes, like inductive ones, serve to establish general rules (from experience) that may be applied to any number of particular cases falling under those rules. Thus, we learn from experience that certain kinds of sensations are signs of certain ideas about distance. Subsequently, when we are stimulated and experience such-and-such retinal sensations, our associative processes lead us to form particular representations of the spatial layout of the world. This analogy is supposed to show that, in both inductive reasoning and visual processing, the associative laws governing the application of general rules to particular cases are based on the "habitual disposition of ideas of type-A [i.e., retinal sensations] to trigger ideas of type-B [i.e., tactile sensations]." 51 This habit is the product of experience. Through experience, we develop the habit of anticipating that

\footnotetext{
${ }^{47}$ Armstrong, quat, 19.

48 Berkeley, An Essay Towardsa NewThearyof Vision 21.

${ }^{49}$ Ibid, 23.

${ }^{50}$ Ibid, 24.

51 Schwartz, qpat, 84.
} 
sensations of type-B will follow from sensations of type-A in a law-like manner. 52

In this sense, Berkeley and Helmholtz claim that visual phenomena have a contingent (a posteriori), rather than a necessary (a priori) connection with distance perceptions. For associationists, there can be no question of deducing the distance of objects from what is immediately given in our visual experience (as Descartes contends), because the relevant cues or signs for depth perception have only a non-deductive connection with the perceived distance of the object in space (i.e., the correlation between ideas of distance and the degree of "ocular strain" felt in our eye-muscles as we bring the object into focus). In other words, we can judge that there will be sensations of type- $B$ when we have sensations of type-A, because in the past we have experienced the conjunction of type-A with type-B. In Berkeley's terminology, tangible perceptions of distance are "suggested" by immediate visual experiences because this connection has been established by constant experience. $\mathrm{He}$ writes, "Having of a long time experienced certain ideas, perceivable by touch, as distance, tangible figure, and solidity, to have been connected with certain ideas of sight, I do upon perceiving these ideas of sight forthwith conclude what tangible ideas are, by the wonted ordinary course of Nature, like to follow." 53 Therefore, whereas Descartes argues for the deductive model of visual processing, Berkeley and Helmholtz contend that there is a contingent, non-deductive connection between our immediate visual experiences and our mediate perceptions of distance.

Despite this disagreement, there is a striking alliance between Descartes, Berkeley and Helmholtz when it comes to the actual problem they choose to address. They agree that distance perception is a cognitive process, mediated by retinal sensations (i.e., that it is indirect). For Descartes and Berkeley, distance perceptions follow experientially from the occurrence of retinal sensations. The disagreements come later on, when the issue is choosing the correct processing model for the job, that is, for specifying those processes involved in going from retinal sensations to distance perceptions. Nevertheless, Descartes and Berkeley (and Helmholtz) concur on the rules of the game.

D epartment of Philosophy, Brock U niversity, Canada

\footnotetext{
${ }^{52}$ As the word "analogy" suggests, neither Berkeley nor Helmholtz is seeking to identify inductive reasoning with visual processing. The two are fundamentally distinct. For example, the associative connection between sensations of type-B with sensations of type-A is not explicitly stated in the symbolic representation "If A then B." In visual processing, the laws governing associative dynamics are automatic and "unconscious" (as Helmholtz puts it). Thus, while the associational model of visual processing is analogous to ordinary inductive reasoning, associative visual processes are not identical with the (inductive) reasoning processes used in logic and science. N either Berkeley nor Helmholtz wishes to conflate the former with the latter.

53 Berkeley, A n E ssay T owards a N ew Theory of V ision, 39.
} 


\section{THE INDIRECT PERCEPTION OF DISTANCE}

\section{References Cited}

Atherton, M., Berkeley's Revolution in V ision (New York: Cornell University Press, 1990).

Armstrong, D.M., Berk eley's Theory of V ision (Australia: Melbourne University Press, 1960).

Berkeley, G., A n E ssay Towards a $\mathrm{N}$ ew Theory of $\mathrm{V}$ ision (New York: BobbsMerrill Company Inc., 1963).

, The Principles of H uman Knowledge (New York: Bobbs-Merrill Company Inc., 1963).

, The Theory of V ision V indicated (New York: Bobbs-Merrill Company Inc., 1963).

Helmholtz, H., T reaties on Physiological 0ptics (New Y ork: D over, 1950).

Schwartz, R., V ision: V ariations on some Berk elean Themes (Oxford and Cambridge U.S.A.: Blackwell Publishers, 1994).

Warnock, G.J., Berk eley (Middlesex England: Penguin Books, 1969). 